\title{
A Quasi-Experiment of Exercise Modality Effects on Cognition and Fitness in Healthy Women
}

\author{
Julie Larsen, Char-Lee Cassel and Judy Knuth \\ Nutrition and Exercise Physiology, Washington State University, Spokane, WA 99210, USA
}

\begin{abstract}
Exercise has been shown to improve cognitive function in many clinical populations. However, there is not a standard exercise prescription for improving cognition among adults. The purpose of this study was to assess the feasibility of standardizing an exercise prescription for cognitive improvement, without reducing fitness benefits of exercise. Healthy women were assigned to a moderately intense floor/step aerobics (FSA; $n=8$ ), treadmill walking (TMW; $n=9$ ), or stretching (STR; $n=10)$ intervention for 7 weeks. Cognition and fitness were assessed pre-, mid-, and post-intervention. FSA participants experienced a significantly reduced time to complete the Trail Making Test-A compared to those in TMW or STR $(P=0.016)$. In conclusion, participation in FSA may result in greater cognitive gains than does participation in TMW, while still providing fitness benefits. For women desiring to improve their cognition and fitness, prescription of moderately intense FSA for 150 minutes each week should be considered.
\end{abstract}

Key words: Cognition, fitness, aerobic exercise.

\section{Introduction}

Participation in exercise has been shown to slow or prevent age-related cognitive decline in numerous studies. A recent study utilized a 6-month intervention for 28 patients with risk factors for cognitive dysfunction [1]. Those who participated in the aerobic exercise intervention displayed significant cognitive improvement on tests of executive function compared to those in the stretching/balance control group (Trail Making Test-B: $P=0.04$; Task Switching: $P=0.03$; Stroop: $P=0.04$ ). Another study demonstrated that a 12-week combined aerobic and strength intervention can significantly improve executive function in a small sample of chronic stroke survivors when measured with the Digit Span Backward test $(P=0.05)$ [2]. It is evident from recent research that participation in regular exercise can enhance cognitive function in a variety of clinical populations.

A specific exercise prescription for cognitive improvement, in terms of frequency, intensity, time,

Corresponding author: Julie Larsen, Ph.D., instructor, research fields: clinical exercise physiology, cognition, and nutrition. and type, has not been established by national organizations. However, there are a few studies that provide insight into exercise programming for cognitive improvement.

\subsection{Frequency}

It has been demonstrated that 3 days of aerobic exercise per week was sufficient to significantly improve self-reported memory in a study of 138 older adults with reported impairments $(P=0.04)$ [3]. In another study of 96 older adults with normal Mini-Mental State Exam scores, 2 days of exercise per week was sufficient to significantly improve processing speed and executive function when compared to a standard care group (Trail Making Test-A and B: $P<0.001$ ) [4]. Therefore, an intervention that occurs 2-3 days per week may enhance a variety of cognitive domains in older adults. Published literature does not indicate whether increased exercise frequency has any additional cognitive benefits.

\subsection{Intensity}

Cognition has been shown to improve following an 
exercise intervention using a variety of workout intensities [4-6]. However, researchers have theorized that cognitive changes follow an inverted U-shaped curve based on exercise intensity, meaning that cognition improves to the greatest extent at moderate intensities, and to a lesser extent at high and low intensities [7]. Therefore, some evidence exists for the benefit of using a moderately intense exercise program.

\subsection{Time}

Several researchers have shown that participation in 45-60 minutes of continuous aerobic exercise can improve cognition $[3,4,6]$. Researchers recruited 21 participants and demonstrated that just 30-minute sessions of aerobic exercise is sufficient to reduce the rate of cognitive decline on the Mini-Mental State Exam in nursing home residents with Alzheimer's disease when compared to a control group (exercise $=$ $13 \%$ decline; control $=47 \%$ decline [5]. This indicates that shorter, more frequent exercise sessions are also beneficial for cognitive health. Workout duration should correspond to workout frequency, so that weekly exercise time of at least 150 minutes is accomplished, which is the minimum exercise volume recommended by the American College of Sports Medicine (ACSM) [8].

\subsection{Type}

Published literature provides little evidence as to which type of exercise is likely to have the greatest impact on cognition. The aforementioned studies primarily utilized Tai Chi [4] and walking [3, 5], showing each to be effective, but none compared different modalities when evaluating improvement in cognition.

Most researchers utilize treadmill walking (TMW) or cycling modalities, but few study the cognitive impact of floor/step aerobics (FSA). One group recently reported that step aerobics can improve functional fitness in elderly women, but it is unknown whether this modality also improves cognition in this population [9]. However, a study of 13 older adults, demonstrated that inclusion of a cognitive stimulus during exercise results in significantly greater cognitive improvements than does listening to music while exercising $(P=0.05)$ [10]. Since FSA involves rapid responses to oral cues, varied movement patterns, and nearly continuous cognitive stimuli, it is proposed that participation in this modality might result in greater cognitive improvement than would participation in an exercise modality that does not involve as many cognitive processes [10].

Since FSA has not been used abundantly in research studies, and it is currently unknown what impact this modality has on cognition, the primary purpose of this study was to determine whether FSA should be recommended to women who desire to improve their cognitive function and aerobic fitness simultaneously. We hypothesized that women who participated in FSA would exhibit greater cognitive improvements than would those who participated in TMW classes. A secondary purpose of this study was to determine whether the FSA modality provides fitness benefits comparable to TMW. If frequency, intensity, and time of exercise are held constant, we hypothesized that there would be an equal improvement in fitness between the two aerobic exercise modalities.

\section{Materials and Methods}

\subsection{Recruitment}

Following Institutional Review Board approval, women were primarily recruited from the Washington State University and the Eastern Washington University shared Riverpoint campus in Spokane through flyers posted around campus and an announcement in the faculty/staff e-newsletter. Advertising materials were distributed to nearby businesses within a 5-minute walking distance from the research lab. An email was also sent to two large employers in Spokane, which was to be distributed to their employees. 


\subsection{Participants}

Inclusion criteria comprised being female and not meeting the ACSM aerobic activity guidelines [8]. Participants were also required to be at "low" or "moderate" risk for adverse events during exercise, as defined by ACSM [8].

Forty-nine women were informed of the procedures for testing and training, and voluntarily signed an informed consent form. The mean age of the women was $48.0 \pm 12.6$ years and mean education was $15.8 \pm$ 2.7 years (mean \pm standard deviation). There were no statistically significant differences in assessed subject characteristics among intervention groups (Table 1).

\subsection{Intervention}

Participants were assigned to a TMW, FSA, or stretching (STR) intervention group based on class time preference. STR was selected as a non-aerobic exercise group to control for improved cognitive scores due to practice effects. Participants underwent seven weeks of exercise training at Washington State University Spokane during the summer. Classes were 50 minutes long and occurred on Mondays, Wednesdays, and Thursdays. Participants were instructed to exercise at a moderate intensity, which was confirmed using $64 \%-74 \%$ of age-predicted maximal heart rate (APMHR), calculated from 220-age and measured with pulse oximetry. Reported ratings of perceived exertion (RPE) from 12-13 on Borg's 6-20 scale were also used to confirm moderate intensity. TMW classes began with 25 minutes of rapid walking, followed by 20 minutes of slower walking at a grade. FSA classes began with 25 minutes of floor aerobics, followed by 20 minutes of step aerobics. Each routine included four 32-count blocks with a variety of upper body and lower body movement patterns. Either a new floor routine or a new step routine was introduced every day, while the other remained the same as the previous day. This allowed participants to have one familiar routine and one new routine each day. Cueing was provided as needed to assist participants in successfully learning the routines. All FSA classes were taught by the same instructor throughout the duration of the study, so cueing remained consistent. Since FSA classes require use of music to maintain the tempo, background music was also played during TMW and STR classes to reduce intergroup variability. All classes contained 5 minutes of warm-up and cool-down.

\subsection{Data Collection}

Participants completed pre-testing (T1: Week -1), mid-testing (T2: Week 4), and post-testing (T3: Week 8) for cognitive function and cardiorespiratory fitness.

An objective cognitive assessment battery was used as a surrogate marker of improvement due to infeasibility of clinical brain function measurements. The cognitive test battery selected consisted of standard tests to assess all primary cognitive domains. The Trail Making Tests A and B (TMT-A and TMT-B) were selected to assess attention, processing speed, and executive function $[11,12]$. The Rey Auditory Verbal Learning Test (RAVLT) was chosen to assess verbal learning and memory [12, 13]. Different word lists were selected for T1, T2, and T3. Per standard protocol, participants were given five immediate recall trials, an interference list, a short-term recall task, and a delayed recall task. Verbal fluency was assessed using the Controlled Oral Word Association Test (COWAT)—FAS [12]. Digit Span Forward (DSF) and Digit Span Backward (DSB) were chosen to assess short-term memory and working memory. Finally, participants completed the North American Adult Reading Test (NAART) as a control for pre-morbid function [12, 14].

Table 1 Participant Demographics (Shown as mean \pm SD).

\begin{tabular}{lllll}
\hline & Aerobics $(n=8)$ & Treadmill $(n=9)$ & Stretching $(n=10)$ & $P$-value \\
\hline Age & $45.0 \pm 13.0$ & $48.4 \pm 13.2$ & $50.3 \pm 12.0$ & 0.501 \\
Education & $15.6 \pm 2.6$ & $15.7 \pm 3.8$ & $16.1 \pm 2.1$ & 0.903 \\
\hline
\end{tabular}


In addition to cognitive testing, participants completed the Ebbeling Submaximal Single-Stage Treadmill Walking Test $[15,16]$ to predict cardiorespiratory fitness levels $\left(\mathrm{VO}_{2}\right)$ at all three time-points. Once participants self-selected their walking speed at $\mathrm{T} 1$, they were required to use that speed for T2 and T3 testing, so the only test variable was exercising heart rate (HR). This submaximal assessment was used as a surrogate prediction of maximal oxygen uptake because actual measurement would have been impractical in terms of cost and participant compliance.

\subsection{Statistical Analyses}

The effect of exercise on cognitive function varies greatly depending on the condition of the participant, the exercise intervention, and the assessment used, among other factors. It has been reported that exercise can have an effect size of 0.3 in certain scenarios [17]. Based on this effect size and a power of 0.80 , it was determined that 25 participants were needed for this study.

Repeated measures ANOVA (SPSS 23) with Bonferroni's post hoc analysis was used to compare the interaction effects of time and intervention group on fitness and cognitive outcomes. Results were considered significant if the $P$-value was $<0.05$. Reported $P$-values are those of the interaction effect of group and time, which indicates whether group assignment affected the level of change over time.

\section{Results and Analysis}

Sixty-eight eligible women contacted the researchers regarding study participation. Forty-nine women completed baseline testing, 33 completed mid-term testing, and 27 completed post-testing. Additional details regarding attrition are shown in Fig. 1.

\subsection{Cognition}

TMT-A and B: There were no significant between-group differences at T1 (TMT-A: $P=0.409$; TMT-B: $P=0.844)$. The intervention resulted in a significant interaction between group and time on education adjusted scores for the TMT-A test $(P=$ 0.016 , partial eta squared $=0.237$, observed power $=$ 0.812), indicating significant improvements in attention and processing speed from FSA when compared to TMW and STR (Fig. 2). There was also a trend toward an interaction between group and time on education-adjustedTMT-B scores $(P=0.301$, partial

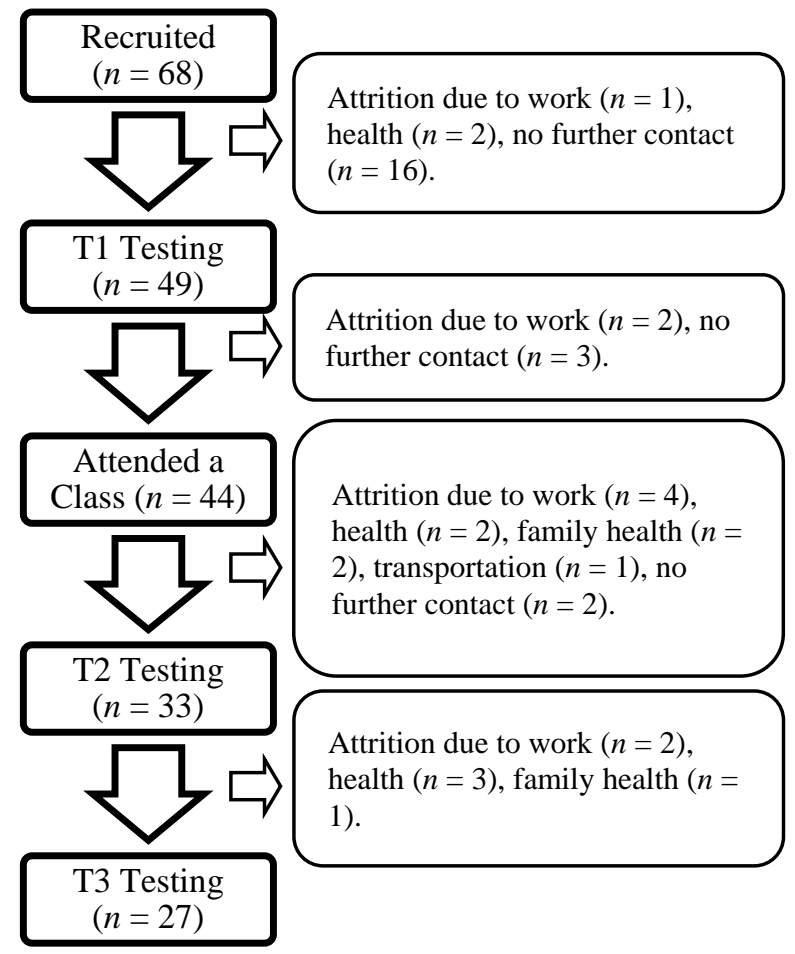

Fig. 1 Participant Recruitment and Attrition.

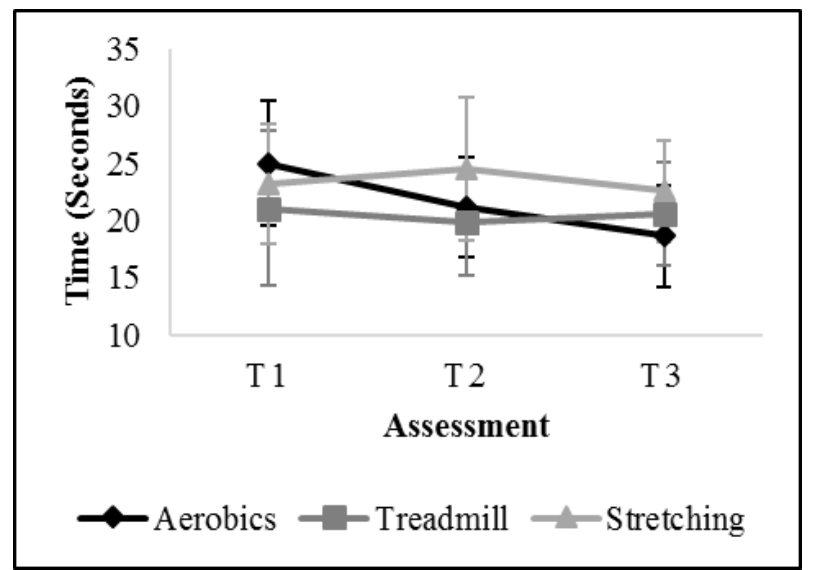

Fig. 2 Effect of exercise modality on Trail Making Test-A (TMT-A) results. 


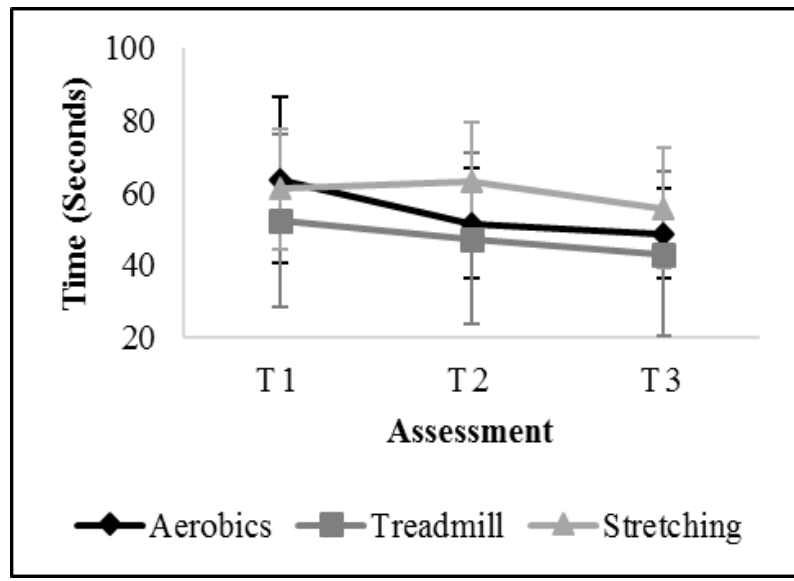

Fig. 3 Effect of exercise modality on Trail Making Test-B (TMT-B) results.

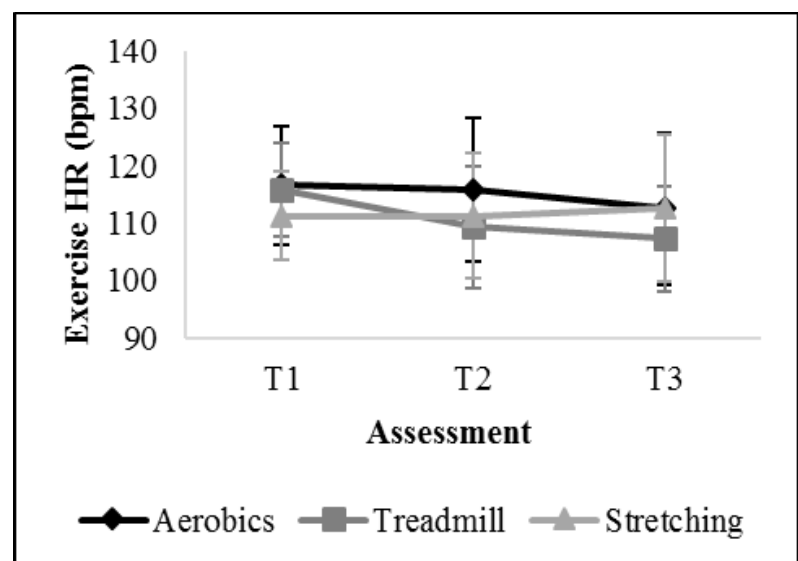

Fig. 4 Effect of exercise modality on exercise heart rate during the Ebbeling Treadmill Test.

eta squared $=0.107$, observed power $=0.359$ ) showing potential improvement in executive function with participation in FSA (Fig. 3).

RAVLT: There were no significant effects of exercise modality over time on verbal learning and memory. Analyses were done for Trial 1 of the RAVLT ( $P=0.729$, partial eta squared $=0.042$, observed power $=0.160)$, Trials $1-5$ total $(P=0.923$, partial eta squared $=0.019$, observed power $=0.094)$, and delayed recall $(P=0.891$, partial eta squared $=0.027$, observed power $=0.105)$. Differences between groups at $\mathrm{T} 1$ were also non-significant (Trial 1: $P=0.752$; Total: $P=0.301$; Delay: $P=0.283$ ).

Digit Span Tests: T1 differences (DSF: $P=0.526$; DSB: $P=0.702)$ and effects of exercise training and modality on DSF $(P=0.794$, partial eta squared $=$
0.035 , observed power $=0.138)$ and DSB $(P=0.740$ with Greenhouse-Geisser correction, partial eta squared $=0.082$, observed power $=0.299$ ) were found to not be significant.

COWAT_FAS: Groups were similar at T1 $(P=$ 0.878 ) and there were no significant interaction effects of exercise modality over time on education-adjusted verbal fluency scaled scores when compared between the three groups $(P=0.251$, partial eta squared $=0.108$, observed power $=0.399)$.

NAART: Although there were no T1 differences between groups $(P=0.495)$, contrary to what was expected, there was an interaction between group and time on NAART scores $(P=0.045$, partial eta squared $=0.195$, observed power $=0.695)$. Since it is unlikely that pre-morbid intelligence was affected by exercise modality, the results are attributed to administrator error.

\subsection{Fitness}

When assessed using predicted maximal $\mathrm{VO}_{2}$ from the Ebbeling treadmill test, there was no difference between groups at $\mathrm{T} 1(P=0.959)$. There was no evidence of fitness improvements from seven weeks of regular participation in FSA, TMW, or STR $(P=0.520$ with Greenhouse-Geisser correction, partial eta squared $=0.064$, observed power $=0.192$ ).

Fitness was also assessed by exercise HR at a given intensity during the Ebbeling test (T1: $P=0.427$ ). Although this analysis did not show significant interaction effects $(P=0.218$, partial eta squared $=$ 0.107 , observed power $=0.430$ ), there was a clear visual trend toward fitness improvements in FSA and TMW, but not in STR, as shown in Fig. 4.

\section{Discussion}

The primary purpose of this study was to determine the feasibility of utilizing FSA as a prescribed aerobic exercise intervention to improve cognitive function and cardiorespiratory fitness in healthy women, as a pilot study for future work with breast cancer survivors. 


\subsection{Cognition}

Previous studies have shown that walking interventions can improve cognition [1, 3,5], but have not studied how FSA impacts cognition. Results of this study demonstrate that seven weeks of FSA, performed three days per week, is sufficient to improve attention and processing speed to a significantly greater extent relative to participation in the same exercise volume on a treadmill. The FSA modality requires participants to quickly decipher commands to alter their movement patterns; TMW is a repetitive modality that does not require many cognitive processes comparatively. Researchers previously reported that having cognitive stimulation while exercising improves cognition more than does exercising without stimuli [10]. Frequent changing of cognitive stimuli is likely associated with the improvement in TMT-A scores among FSA participants.

It was anticipated that executive function and short-term memory would also improve following an exercise intervention. The multi-tasking ability and memory required for successfully completing FSA should improve these related brain functioning processes. However, factors that contributed to the failure to demonstrate significant benefits to executive function and short-term memory may include the following: the intervention was not long enough in duration, the routines were not adequate to stimulate these cognitive improvements, the assessments were not sensitive enough to detect cognitive changes, and/or the study was under-powered.

\subsection{Fitness}

Theoretically, any moderately intense exercise intervention should improve cardiorespiratory fitness. However, the equation for predicting maximal $\mathrm{VO}_{2}$ from Ebbeling test results did not demonstrate substantial fitness improvements, because exercising HR is included as a factor in both an addend and a subtrahend. Treadmill speed was held constant from T1 to $\mathrm{T} 3$, as an attempt to reduce variability, but may have invalidated the test since it is validated at a "self-selected" speed and participants were not allowed to select their speed after T1. As their fitness improved, they likely would have selected a faster speed, which would have resulted in a higher $\mathrm{VO}_{2}$ max calculation.

Fortunately, it was still possible to assess fitness changes with exercise modality. As cardiorespiratory fitness increases, heart rate at a given intensity will be lower. A similarly designed post-testing protocol was used in a previously published study [18]. In this study, the results demonstrated improved fitness, based on exercising $\mathrm{HR}$ at a given intensity, following an exercise intervention. Based on our results, in which HR decreased at the same treadmill speed from T1 to T3, there was similar trend toward improvement in fitness between the FSA and TMW groups. The slightly greater improvement in TMW was likely due to the Ebbeling treadmill test replicating the TMW intervention.

\subsection{Limitations}

Limited staffing prevented the researchers from being blinded to exercise interventions. Those who taught exercise classes also conducted pre-, mid-, and post-testing, introducing the possibility of administrator bias. In addition, the staff, while trained and prepared, were inexperienced at administering cognitive tests. As they gained more experience, they may have subconsciously altered their scoring methods. As mentioned previously, this could have invalidated the NAART results because that assessment is scored subjectively. All other cognitive assessments that were used are scored objectively (time to completion, length of number series recalled, number of words recited, etc.), reducing the negative impact of inexperience on validity of administration. Another limitation to this study was attrition. Nearly half of those who were tested at T1 dropped out before T3. Due to the high attrition rate, in future studies we recommend an incentive for participants after completion of post-testing. Finally, APMHR has a relatively high 
degree of inaccuracy, limiting its usefulness in confirming adherence to prescribed exercise intensity. RPE is also limited in usefulness due to its subjectivity. However, dual-confirmation of moderate intensity utilizing these two methods enhances validity.

\section{Conclusions}

FSA is an acceptable modality for improving cognition and fitness among healthy adult middle-aged to elderly women. By assessing HR at least twice per session, participants were generally able to remain within a moderately intense exercise range. FSA classes are relatively easy to modify for either increased or decreased intensity needs without singling out participants who have lower fitness levels.

The FSA exercise modality results in greater cognitive improvement than does TMW. Changes in cognitive test results, with regard to exercise modality, were significant on the TMT-A and trended towards significance on the TMT-B. This information is particularly critical when working with older women, those concerned about age-related cognitive decline, and those at increased risk for cognitive dysfunction.

Future studies of similar design should include a non-exercise control group, as opposed to STR, to further establish the cognitive benefits of aerobic exercise modalities. A longer intervention, greater class frequency, and/or higher exercise intensity might elicit greater cognitive benefits than were reported here. More research is needed in this field to solidly determine an exercise prescription to improve cognition and fitness simultaneously.

\section{Acknowledgment}

Funding for this study was provided by a College of Pharmacy Seed Grant from Washington State University.

\section{References}

[1] Baker, L. D., Frank, L. L., Foster-Schubert, K., Green, P. S., Wilkinson, C. W., McTiernan, A, Cholerton, B. A., Plymate, S. R., Fishel, M. A., Watson, G. S., Duncan, G.
E., Mehta, P. D., and Craft, S. 2010. “Aerobic Exercise Improves Cognition for Older Adults with Glucose Intolerance, a Risk Factor for Alzheimer's Disease." Journal of Alzheimer's Disease 22 (2): 569-79.

[2] Kluding, P. M., Tseng, B. Y., and Billinger, S. A. 2011. "Exercise and Executive Function in Individuals with Chronic Stroke: A Pilot Study." Journal of Neurologic Physical Therapy 35 (1): 11-7.

[3] Lautenschlager, N. T., Cox, K. L., Flicker, L., Foster, J. K., van Bockxmeer, F. M., Xiao, J., Greenop, K. R., and Almeida, O. P. 2008. "Effect of Physical Activity on Cognitive Function in Older Adults at Risk for Alzheimer Disease: A Randomized Trial." Journal of the American Medical Association 300 (9): 1027-37.

[4] Nguyen, M. H., and Kruse, A. 2012. “A Randomized Controlled Trial of Tai Chi for Balance, Sleep Quality and Cognitive Performance in Elderly Vietnamese." Clinical Interventions in Aging 7: 185-90.

[5] Venturelli, M., Scarsini, R., and Schena, F. 2011. "Six-Month Walking Program Changes Cognitive and ADL Performance in Patients with Alzheimer." American Journal of Alzheimer's Disease \& Other Dementias 26 (5): 381-8.

[6] Baker, L. D., Frank, L. L., Foster-Schubert, K., Green, P. S., Wilkinson, C. W., McTiernan, A., Plymate, S. R., Fishel, M. A., Watson, G. S., Cholerton, B. A., Duncan, G. E., Mehta, P. D., and Craft, S. 2010. "Effects of Aerobic Exercise on Mild Cognitive Impairment: A Controlled Trial." Archives of Neurology 67 (1): 71-9.

[7] Kamijo, K., Nishihira, Y., Higashiura, T., and Kuroiwa, K. 2007. "The Interactive Effect of Exercise Intensity and Task Difficulty on Human Cognitive Processing." International Journal of Psychophysiology 65 (2): 114-21.

[8] Pescatello, L. S., Arena, R., Riebe, D., and Thompson, P. D. ed. 2014. ACSM's Guidelines for Exercise Testing and Prescription, 9th ed. Philadelphia: Wolters Kluwer Health; Lippincott Williams \& Wilkins.

[9] Hallage, T., Krause, M. P., Halie, L., Miculis, C. P., Nagle, E. F., Reis, R. S, and Da Silva, S. G. 2010. "The Effects of 12 Weeks of Step Aerobics Training on Functional Fitness of Elderly Women." Journal of Strength \& Conditioning Research 24 (8): 2261-6.

[10] You, J. H., Shetty, A., Jones, T., Shields, K., Belay, Y., and Brown, D. 2009. "Effects of Dual-Task Cognitive-Gait Intervention on Memory and Gait Dynamics in Older Adults with a History of Falls: A Preliminary Investigation." NeuroRehabilitation Journal 24 (2): 193-8.

[11] Staff, Personnel Research Section, Classification and Replacement Branch, AGO. 1944. "The New Army Individual Test of General Mental Ability.” Psychological 
Bulletin 41 (8): 532-8.

[12] Lezak, M. 1983. Neuropsychological Assessment. New York: Oxford University Press.

[13] Rey, A. 1964. The Examination in Clinical Psychology. Paris: Press Universitaire de France. (in French)

[14] Blair, J., and Spreen, O. 1989. "Predicting Premorbid IQ: A Revision of the National Adult Reading Test." Clinical Neuropsychologist 3 (2): 129-36.

[15] Mitros, M., Gabriel, K. P., Ainsworth, B., Lee, C., Hermann, S., Campbell, K., and Swan, P. 2011. "Comprehensive Evaluation of a Single-Stage Submaximal Treadmill Walking Protocol in Healthy, Middle-Aged Women." European Journal of Applied
Physiology 111 (1): 47-56.

[16] Waddoups, L., Wagner, D., Fallon, J. and Heath, E. 2008. "Validation of a Single-Stage Submaximal Treadmill Walking Test." Journal of Sports Science 26 (5): 491-7.

[17] Angevaren, M., Aufdemkampe, G., Verharr, H. J., Aleman, A., and Vanhees, L. 2008. "Physical Activity and Enhanced Fitness to Improve Cognitive Function in Older People without Known Cognitive Impairment." Cochrane Database System Reviews 3 (2): 1-73.

[18] Pinto, B. M., Clark, M. M., Maruyama, N. C. and Feder, S. I. 2003. "Psychological and Fitness Changes Associated with Exercise Participation among Women with Breast Cancer." Psychooncology 12 (2): 118-2. 